\title{
Mal de Pott cervical révélé par une dysphagie: À propos d'un cas.
}

\section{Cervical Pott's disease revealed by dysphagia: a case report.}

Kodio B ${ }^{\mathbf{1}, 2}$, Cissé $\mathrm{H}^{\mathbf{3}}$, ETI E ${ }^{\mathbf{1}}$, Ouattara Baly ${ }^{1}$, Koné Gbané $\mathrm{M}^{\mathbf{1}}$, Kouakou N'zué $\mathrm{M}^{\mathbf{1}}$.

1 Service de Rhumatologie, CHU de Cocody-Côte D'Ivoire

2 Service de Rhumatologie, CHU du Point G, Bamako Mali

3 Service de Maladies infectieuses, Hôpital de Sikasso, Mali

correspondance :Dr Boureima KODIO, Rhumatologue. Email : boureimakodio@gmail.com

\section{Résumé :}

La tuberculose est encore endémique dans nos contrées et de modes de révélation multiples. Le Mal de Pott cervical est la localisation rachidienne la plus rare. La compression médullaire, les abcès rétropharyngés et épiduraux en sont des complications graves pouvant engager le pronostic. Nous rapportons un cas de Mal de Pott cervical révélé par une dysphagie due à un abcès rétropharyngé ; chez un patient de 34 ans ayant été victime d'effraction de l'oropharynx par une arête de poisson. Cette observation nous révèle l'une des facettes de la tuberculose.

Mots Clés : Arête de poisson, Dysphagie, Tuberculose cervicale.

\section{Summary:}

Tuberculosis is endemic in our countries and multiple modes of revelation. The cervical Pott's disease is the rarest spinal location. Spinal cord compression, retropharyngeal and epidural abscess are serious complications that can engage the prognosis. We report a case of cervical Pott's disease revealed by dysphagia due to a retropharyngeal abscess in a patient of 34 years has been the victim of the oropharynx injury by a fish bone. This observation reveals one of the many facets of tuberculosis.

Keywords: Cervical Tuberculosis, Dysphagia, Fish bone.

\section{Introduction}


La spondylodiscite cervicale représente la localisation tuberculeuse aux cinq dernières vertèbres cervicales. Elle se distingue des autres localisations rachidiennes par sa rareté (2 à $10 \%$ ) $[1,2]$. Les abcès épidural et rétropharyngé, la compression médullaire en sont les complications graves qui engagent le pronostic. L'abcès rétropharyngé est la plus rare des suppurations péripharyngées et le souvent secondaire à l'ingestion d'un corps étranger ou à un traumatisme de la paroi pharyngée postérieure [3]. Nous rapportons un cas de tuberculose cervicale révélée par une dysphagie.

\section{Observation}

A. D patient de 34 ans, livreur de pain, a eu une notion d'effraction de l'oropharynx par une arête de poisson en 2012 pendant qu'il mangeait. Il a consulté en janvier 2014 en ORL pour une dysphagie accompagnée d'odynophagie et de dysphonie. L'examen a mis en évidence une tuméfaction de l'oropharynx, et le diagnostic évoqué a été celui d'un abcès rétropharyngé. Le traitement a comporté une ponction évacuatrice de l'abcès, une antibiothérapie à base d'amoxicilline + acide clavulanique et métronidazole pendant 15 jours; mais devant l'apparition d'une cervicalgie inflammatoire avec névralgie cervicobrachiale bilatérale, mal systématisée et des acroparesthésies, un scanner du rachis cervical réalisé qui objectivera une spondylodiscite étagée C3-C4-C5-C6. Devant ce tableau le patient est adressé en rhumatologie ou il sera hospitalisé en février 2014. L'interrogatoire a trouvé un contexte de fièvre vespérale, d'asthénie et de perte pondérale non quantifiée. A l'examen, l'état général était altéré. La température était à $38,2^{\circ} \mathrm{C}$. L'examen ostéoarticulaire a objectivé un syndrome rachidien cervical marqué, une raideur cervicale invincible, un syndrome pyramidal des 4 membres avec des réflexes ostéo-tendineux vifs, la présence des signes de Babinski et de Hoffmann, sans déficit neuromusculaire. Sur le plan paraclinique on notait une élévation de la VS à $48 \mathrm{~mm}$ à la $1^{\text {ere }}$ heure et de la CRP à $27,1 \mathrm{mg} / \mathrm{l}$. L'hémogramme était normal. Il n'y avait pas d'hyperleucocytose. La recherche bactériologique (germes banals et BK) était négative sur 3 prélèvements de pus. L'intradermoréaction à la tuberculine était négative de même que la sérologie VIH. La biopsie discovertébrale n'a pas pu être effectuée. La radiographie pulmonaire était sans anomalie. Un deuxième scanner du rachis cervical est réalisé, confirmant la spondylodiscite pluri-étagée avec une ostéolyse du mur postérieur de C3, des margelles antérieures de C5 et C6, un tassement de C4, associée à une image d'abcès épidural compressif et d'un volumineux abcès rétropharyngé sténosant. (Figure 1 et Figure 2). Le diagnostic de spondylodiscite cervicale 
tuberculeuse probable avec épidurite compressive et abcès rétropharyngé a été retenu en l'absence de preuve anatomopathologique devant les arguments clinique et radiologique en zone d'endémie. L'attitude thérapeutique a consisté à :

- Un drainage de l'abcès rétropharyngé,

- Un traitement antituberculeux 2RHZE/10RH : Rifampicine $150 \mathrm{mg}$, Isoniazide $75 \mathrm{mg}$, Pyrazinamide $400 \mathrm{mg}$ et Ethambutol 75 mg en comprimé (forme combinée).

- Une corticothérapie à 40 mg/jour de Prednisone pendant 15 jours.

- Une immobilisation cervicale à l'aide d'un collier cervical rigide.

Un avis neurochirurgical a été demandé et l'indication de corporectomie avec greffe et ostéosynthèse a été posée. Cependant l'intervention n'a pas pu être réalisée à cause du coût onéreux pour le patient.

L'évolution clinique à 4 mois était marquée par la reprise d'une déglutition et d'une phonation normales, et la disparition des cervicalgies et des signes neurologiques. À 6 mois on notait une régression importante des signes radiographiques.

\section{Discussion}

La tuberculose rachidienne est encore fréquente en Afrique noire mais sa localisation cervicale demeure rare [1,2]. La spondylodiscite cervicale dont le maitre symptôme est la cervicalgie, peut être révélée par un abcès rétropharyngé comme premier signe d'appel [3], dont la dysphagie, la dysphonie ou l'odynophagie en sont les prodromes. L'abcès pottique rétropharyngé est exceptionnel. Il est le plus souvent secondaire à l'ingestion d'un corps étranger ou à un traumatisme de la paroi pharyngée postérieure [3]. Notre patient rapporte une effraction de l'oropharynx par une arête de poisson survenue il y a environ deux ans. Quels sont les rapports entre l'arête de poisson, l'effraction de l'oropharynx et le Mal de Pott? C'est tout l'intérêt de notre observation. Le scanner du rachis cervical a montré une spondylodiscite pluri-étagée avec une ostéolyse du mur postérieur de $\mathrm{C} 3$, des margelles antérieures de C5 et $\mathrm{C} 6$, un tassement de $\mathrm{C} 4$, associée à un abcès épidural compressif et un volumineux abcès rétropharyngé. Les lésions radiologiques si évoluées suggèrent que la maladie évolue depuis un certain temps, ou bien que le germe soit très virulent. La première hypothèse semble être le cas chez notre patient chez qui le traumatisme de l'oropharynx remonte à plus de 2 ans.

Le diagnostic de certitude de tuberculose vertébrale est laborieux dans notre contexte. En effet la mise en évidence du BK au niveau du 
foyer pottique étant difficile comme l'affirment d'autres auteurs tels Badr et al [4], l'examen anatomopathologique prend une importance capitale. Mais celui-ci n'a pu être effectué à cause du coût onéreux de la biopsie chirurgicale. Ainsi le diagnostic est le plus souvent retenu sur des arguments radiocliniques et les épreuves thérapeutiques comme l'attestent de nombreux auteurs [5, 6]. Dans les zones d'endémie tuberculeuse, on se contente d'un diagnostic de présomption pour démarrer les antituberculeux [4], vu que le Mal de Pott cervical en l'occurrence est une urgence médicale [7]. Il pose un problème thérapeutique du fait de la complexité anatomique du rachis cervical. Notre patient a bénéficié d'un traitement antituberculeux (2RHZE/10RH) selon le protocole en vigueur en Côte d'Ivoire, une corticothérapie par Prednisone à 40 $\mathrm{mg} /$ jour pendant 15 jours et une immobilisation cervicale par un collier cervical rigide. L'abcès rétropharyngé a été drainé. L'attitude thérapeutique devant un Mal de Pott continue d'être partagée entre le traitement médical exclusif et le traitement médico-chirurgical. Selon Debeyre et al [8] les traitements antituberculeux doivent être administrés à dose maximale d'emblée et sous forme d'une polychimiothérapie associant au moins trois antituberculeux; pour éviter toute résistance au $\mathrm{BK}$, de façon continue et prolongée. La durée du traitement varie entre 6 et 18 mois. Cependant en cas d'atteinte ostéoarticulaire la durée minimale du traitement doit être de 12 mois. L'immobilisation plâtrée reste un complément thérapeutique classique chez tous les auteurs [4]. Elle est utile pour calmer des douleurs intenses ou éviter une déformation consécutive à un tassement vertébral. Les abcès épiduraux et rétropharyngiens peuvent cependant être drainés surtout s'ils sont volumineux afin d'accélérer la guérison [9]. L'évolution clinique favorable sous traitement médical est la règle en quelques semaines. $\mathrm{La}$ guérison radiologique se fait en général vers la constitution d'un bloc osseux solide obtenu en moins d'un an avec une persistance des images de destruction osseuse [10]. Avec un recul de 8 mois, nous avons noté une efficacité thérapeutique. Ceci est un argument supplémentaire en faveur de notre diagnostic.

\section{CONCLUSION :}

L'intérêt de cette observation réside dans son mode de début marquée par une effraction de l'oropharynx par une arête de poisson et des signes laryngés au premier plan. Le diagnostic de tuberculose reste présomptif en l'absence d'une preuve anatomopathologique ou bactériologique.

\section{Conflits d'intérêt : Aucun.}




\section{Références}

1. Alliez JR, Balan C, Diallo O et al. Pott's disease and Pott's paraplegia. AJNS. 2006; 25(2): 50-9.

2. Alifdal M, El Abbadi N, Tamehmacht $\mathrm{M}$ et al. Mal de Pott cervical: considérations thérapeutiques à propos de 11 cas. Rev Mar Chir Orthop Traum 1995 ; $5: 18-22$.

3. Baili L, Rachdi I, Ben DhaouB et al. Mal de Pott cervical révélé par un abcès rétropharyngé. Rev Med Int 2011; 32S: S99-S191

4. Badr F, Khalid C, El Faiz Chaoui M. Le Mal de Pott: à propos de 82 cas. Pan Afr Med J. 2011; 8:22.

5. Loembe PM, Assengone-Zeh Y, Guerch $M$ et al. La tuberculose vertébrale au Gabon. Neurochirurgie. 1988;34(6):420-7.
6. Camara P, Sané M, Margery J et al. Le Mal de Pott dans un service de Médecine interne à l'Hôpital principal de Dakar, étude rétrospective sur 3 ans. Med Trop 2004 ; 64 (3): 270.

7. Journeau $\mathrm{P}$, Haumont $\mathrm{T}$. Cervicalgies aiguës de l'enfant. Rev Rhum 2004 ; 71 : 670-82

8. Debeyre J. Mal de Pott, traitement médical ou médico-chirurgical Table ronde $49 \mathrm{e}$ réunion annuelle SOFCOT. Rev Chir Orthop. 1975; 61(1): 133-340

9. Boussel L, Marchand B, Blineau $\mathrm{N}$ et al. Imagerie de la tuberculose ostéoarticulaire .J Radiol (2002); 83 :1025-34.

10. Zlitini M, Kassab MT. Spondylodiscite tuberculeuse (Mal de Pott). Encyclo Med-Chir App Locomoteur. 1988; 15852: A10 $14 \mathrm{P}$ 


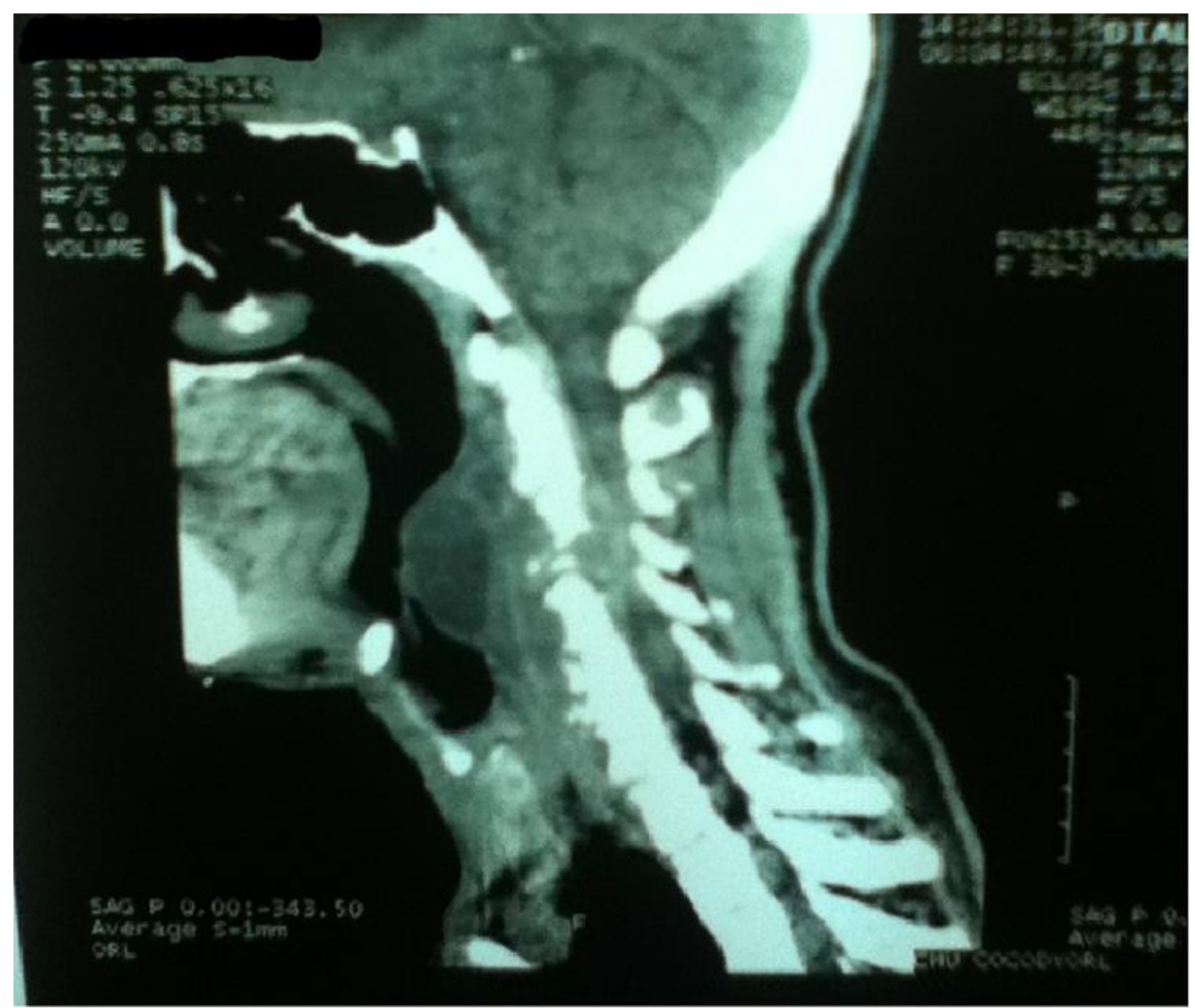

Figure 1: Tomodensitométrie cervicale, Abcès épidural compressif. 


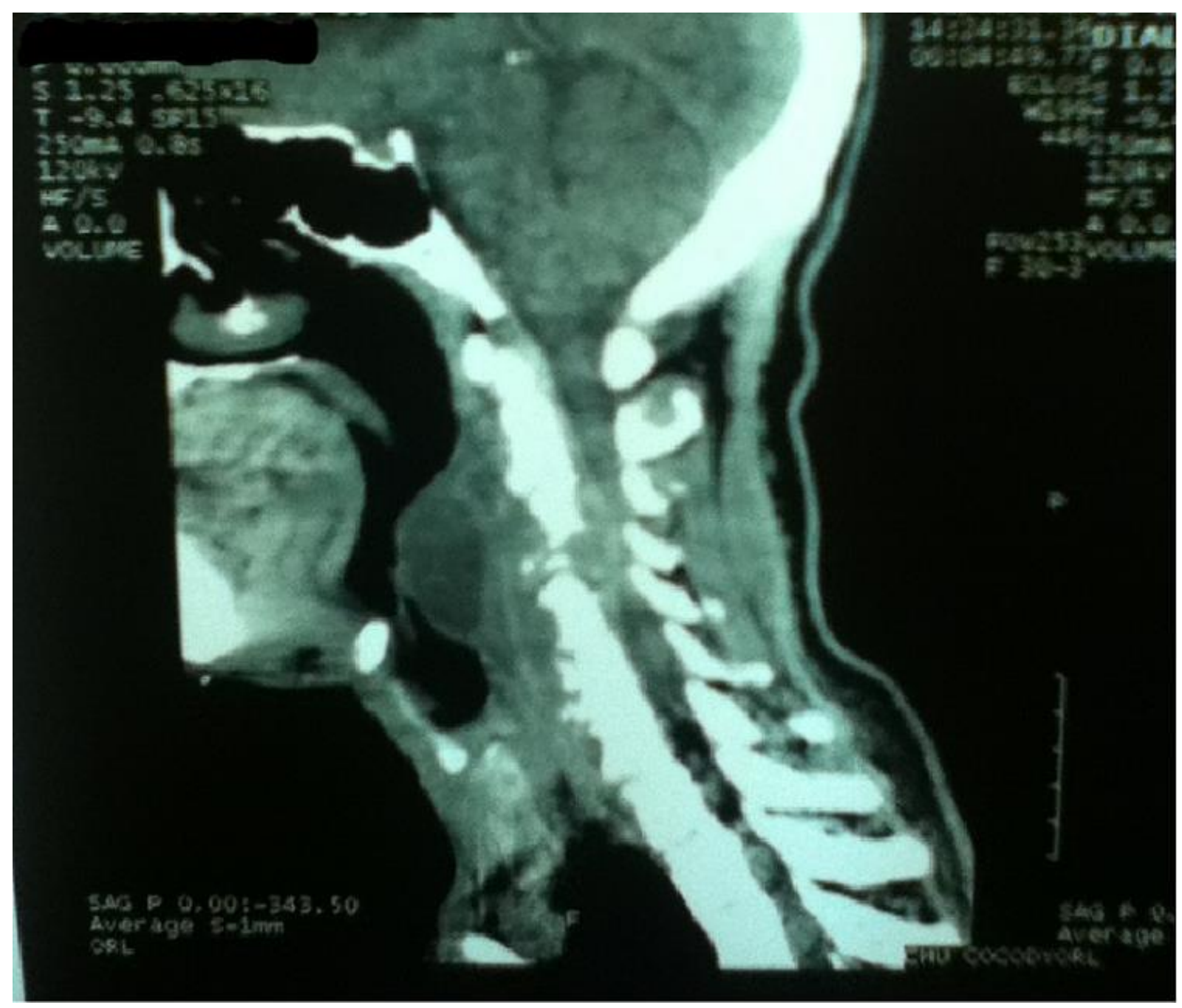

Figure 2: Tomodensitométrie cervicale. Abcès rétropharyngé avec sténose de la lumière œsophagienne 